Rabaska

Revue d'ethnologie de l'Amérique française

\title{
Association des étudiants(e)s en ethnologie du Québec
}

\section{Marie-Christine Gagnon et Mona-Élise Sévigny}

Numéro 1, 2003

URI : https://id.erudit.org/iderudit/201639ar

DOI : https://doi.org/10.7202/201639ar

Aller au sommaire du numéro

Éditeur(s)

Société québécoise d'ethnologie

ISSN

1703-7433 (imprimé)

1916-7350 (numérique)

Découvrir la revue

Citer ce document

Gagnon, M.-C. \& Sévigny, M.-É. (2003). Association des étudiants(e)s en

ethnologie du Québec. Rabaska, (1), 227-228. https://doi.org/10.7202/201639ar d'utilisation que vous pouvez consulter en ligne.

https://apropos.erudit.org/fr/usagers/politique-dutilisation/ 


\section{Association des étudiants(e)s en ethnologie du Québec}

Pavillon Charles-De Koninck, local 0221 Courriel : aeeq@hotmail.com Université Laval

Sainte-Foy (Québec) G1K 7P4

L'Association des étudiants(e)s en ethnologie du Québec a évolué au rythme des changements disciplinaires survenus à l'université Laval. Cette année, elle a connu un réaménagement suite à l'instauration du nouveau programme de baccalauréat intégré en anthropologie et ethnologie. Ainsi, les préoccupations des étudiants membres visent à assimiler les nouvelles orientations que prend l'ethnologie dans le cadre universitaire.

Les principaux objectifs de la nouvelle association sont d'abord de rassembler et de créer un sentiment d'appartenance des étudiants vis-à-vis 
du programme et du corps professoral. Ensuite, elle s'est donnée comme mission de faire connaître l'ethnologie non seulement à l'intérieur de l'université mais aussi auprès d'un plus large public. Évidemment, l'Association est aussi la tribune des étudiants qui désirent mettre de l'avant les choses à améliorer pour assurer le développement positif des études en ethnologie à l'université. De cette façon, l'Association tend à devenir l'intermédiaire entre ses membres et l'université. Pour y parvenir, elle maintient un lien solide avec les professeurs.

Par ailleurs, puisque le besoin se fait sentir de voir ce qui se passe à l'extérieur du milieu universitaire, les étudiants tentent de se rapprocher de différents organismes dont la promotion est largement faite par les professeurs, par exemple le Centre de valorisation du patrimoine vivant et la Société québécoise d'ethnologie. De plus, la journée « Rencontre avec l'ethnologie » et autres colloques sont l'occasion pour l'Association d'entrer en contact avec des ethnologues professionnels et d'assurer une visibilité et une crédibilité au programme.

Les étudiants représentés par l'Association des étudiant(e)s en ethnologie du Québec sont inscrits soit au nouveau programme intégré mentionné plus haut, soit au certificat ou au diplôme en ethnologie. Les étudiants des $2^{\mathrm{e}}$ et $3^{\mathrm{e}}$ cycles font aussi partie des gens que représente l'Association quoique de façon plus distante étant donné les différences académiques entre les niveaux. Le nouveau président, Jocelyn Gadbois, est en troisième année du baccalauréat intégré. La vice-présidente aux communications et aux activités socioculturelles, Marie-Christine Gagnon, termine le certificat en ethnologie et sera à la maîtrise en septembre prochain. Le trésorier, Patric Quirion, et la secrétaire, Mona-Élise Sévigny, commencent tous deux leur deuxième année au baccalauréat intégré. Le poste de représentant aux affaires externes est actuellement vacant.

Durant cette année de changements, l'Association a tout de même tenu quelques activités de rassemblement : souper étudiants-professeurs au bar Oxygène, partie de sucre et mise en place d'un petit comité de l'album pour les deux finissantes de cette année. Actuellement, nous sommes à préparer 1 'initiation des nouveaux arrivants en septembre 2003. L'événement promet d'être joyeux et coloré ! Pour toute question, transmission de message aux étudiants ou commentaires, n'hésitez pas à contacter l'Association.

MARIE-Christine GAGNON MoNA-ÉLISE SÉvigNY 